Result. All respondents $(\mathrm{n}=10)$ dealt with confidentiality issues at work, with $50 \%$ experiencing issues daily. $33 \%$ respondents did not feel confident dealing with confidentiality queries at work. The majority (60\%) had received confidentiality training, but all respondents thought extra information would be useful. Of possible interventions, 70\% supported a flowchart. Following an implementation period, 100\% respondents re-surveyed agreed they felt confident dealing with issues related to confidentiality at work. The majority of respondents had used the flowchart and found it useful (83\%). Qualitative data gathered suggested rollingout the project elsewhere.

Conclusion. A lack of confidence surrounding issues with confidentiality, including information sharing, was identified. This can negatively impact patient engagement and delivery of care. The introduction of the confidentiality flowchart demonstrated improved understanding of, and confidence in, patient confidentiality issues. The small sample size means there are limitations in extrapolating findings to wider contexts. However, it is likely that more confidentiality training and practical information for NHS staff at the interface between patients, clinicians and services would reduce the risk of confidentiality breaches and reinforce positive relationships with services.

Improving patient access to medication information: a quality improvement project on patient-centred prescribing

Anna Todd* and Rosy Blunstone

Bethlem Royal Hospital, South London and Maudsley NHS

Foundation Trust

${ }^{*}$ Corresponding author.

doi: 10.1192/bjo.2021.600

Aims. Ward rounds are sometimes the only opportunity for patients to discuss medication. Patient and professional feedback on an acute male inpatient ward in South London highlighted a demand for more medication information outside the formal ward round setting. We aimed to have $100 \%$ of patients meet our criteria for "Patient-Centred Prescribing" on the ward by March 2021. To fulfil criteria, all patients are offered: (1) ward round discussion, (2) written patient information leaflets (PILs), (3) informal discussion groups, all regarding medication.

The principles of this quality improvement project (QIP) were drawn from definitions of patient-centred care and standards of good practice; patients should have access to a variety of information formats, relevant to the individual, and the knowledge gained empowers patients.

Patient experience data revealed that $30 \%$ of clients answered passively to the question, "Do you feel involved in your care?" We hypothesized that medication discussion groups positively impact patients' wellbeing, by providing a safe space that facilitates conversation surrounding medication issues.

Method. We conducted weekly audits on patients whose admission duration was $>7$ days, and recorded fulfilment of the above criteria. At week 1, we introduced a program of weekly medication discussion groups led by members of the wider multidisciplinary team covering a broad topic range. At week 6 , we developed a rolling rota of the discussion groups and posters were displayed in advance. At week 14, all patients were offered PILs through a 1:1 interaction and this continued as routine practice. Medication discussion group feedback was obtained via questionnaires and "The Blob Tree", a psycho-emotional assessment tool commonly used in healthcare settings.
Result. In 19 weeks, the median percentage of patients who fulfilled our criteria for Patient-Centred Prescribing was 92.86\%. After 11 medication discussion groups, $79.3 \%$ of questionnaire responders wanted further sessions. $88 \%$ of "The Blob Tree" responses collected inferred a positive emotional response after the group discussions and half of those noticed an improvement in their emotional state. Conclusion. This QIP was overall a success; it fulfilled a requirement to meet good standards in information sharing and became embedded in the fabric of the ward, continuing to run as part of the activities program. It demonstrated the impact of education on patients' mental wellbeing through empowerment and peer support. As a by-product it established multidisciplinary connections and improved therapeutic relationships. Challenges included patient engagement secondary to acute mental illness or negative symptoms and maintaining project momentum following a COVID-19 outbreak.

\section{On-line memory clinic - piloting a hybrid model}

\section{Rahul Tomar}

Logandene, Hertfordshire Partnership University NHS Foundation NHS Trust

doi: 10.1192/bjo.2021.601

Aims. Quality improvement project was undertaken to reorganise memory clinic to incorporate both virtual and in-person consultation (Hybrid Virtual model), as depicted in the following model: Method. Tele triage conducted to ascertain information from patient and carer. This reduced time for face to face assessment.

Nurse did face to face assessment to complete cognitive test (Addenbrooke's Cognitive Examination III) \& carer completed Bristol Activities of Daily Living scale. Nurse would also do BP, $\mathrm{PR}$, oxygen sats \& temp.

Nurse discussed the assessment with the consultant (who is in the inpatient unit) on line using MS TEAMS

Consultant would then see patient on line, confirm diagnosis, answer questions, give information on medication and post prescription (if required)

Feedback was collected using Telehealth Satisfaction questionnaire Result. Hybrid remote memory clinic was started on 29/09/20. A total of 37 patients were seen in this clinic by 31/01/21.

Collected feedback from 21 patients was generally positive Information provided on video consultation prior to assessment -18 reported it as excellent

How well you privacy was respected - 21 reported it as excellent. Information you received on the treatment -18 reported it as excellent

Conclusion. The hybrid remote memory clinic was more effective than telephone consultation or on line only consultation as it was -

Easy to establishing rapport

Physical examination could be performed

Digital literacy was no longer a limiting factor

Prescribing medication was slightly more difficult but possible

Audit of follow-up within 7 days on discharge from the mental health unit, Forth Valley Royal Hospital

Matthew Turner ${ }^{1 *}$, Shaun Love ${ }^{2}$, Fergus Douds ${ }^{1}$

and Anyssa Zebda ${ }^{1}$

${ }^{1}$ Forth Valley Royal Hospital and ${ }^{2}$ NHS Greater Glasgow and Clyde ${ }^{\star}$ Corresponding author.

doi: 10.1192/bjo.2021.602 
Aims. To determine compliance with the new discharge policy of review within 7-days for all General Adult Psychiatry patients discharged from Forth Valley Royal Hospital.

Background. It is well established that there is an increased risk of suicide following discharge from Inpatient Psychiatric Wards. This risk is significantly increased in the first month, and particularly high in the first week.

In their 2016 Guidance, NICE recommends follow-up within 7 days of discharge. It is not known whether seven day follow-up reduces suicide risk but it is clearly an opportunity for risk assessment and management during a particularly risky period.

This standard was adopted by the General Adult Wards in Mental Health Unit at Forth Valley Royal Hospital in April 2019. Method. All discharges from Wards 1, 2 and 3, Forth Valley Royal Hospital were reviewed during three distinct, month-long periods:

November 2018 (prior to the introduction of the new discharge policy)

May 2019 (shortly after the introduction of the new discharge policy)

September 2019 (six months after the introduction of the new discharge policy)

A list was obtained from Medical Records of all General Adult patients discharged in these periods. The paper and electronic records were checked for each patient, and the first scheduled care episode post discharge was taken as follow-up.

Result. In the1st round of audit (November 2018): 41 patients were discharged and 26 patients (64\%) received follow-up within 7 days.

In the 2nd round of audit (May 2019): 46 patients were discharged, 39 patients (84\%) received follow-up within 7 days.

In the 3rd round of the audit (September 2019), 50 patients were discharged and 49 (98\%) received follow-up within 7 days. Conclusion. There has been a clear improvement in the provision of follow-up on discharge from the General Adult Psychiatry Wards in Forth Valley Royal Hospital.

The new discharge policy was implemented in April 2019 and a "Discharge Pause" was introduced (initially a sticker, now an electronic form) to be completed by the medical team at the point when it was decided to discharge.

Community Mental Health Teams have also been reminded of their need to facilitate seven day follow-up as a priority. A flowchart was produced in May 2019, which provided guidance as to who should provide the seven day follow-up.

\section{Professional training and case-load mix within a community mental health team}

Richard Walsh ${ }^{1 \star}$, Rebecca Fahy ${ }^{2}$, Ala Abdelgadir ${ }^{3}$, Elizabeth Walsh ${ }^{3}$ and Sonn Patel ${ }^{3}$

${ }^{1}$ School of Medicine, University College Dublin; ${ }^{2}$ Semmelweis University and ${ }^{3}$ Galway University Hospital

${ }^{\star}$ Corresponding author.

doi: 10.1192/bjo.2021.603

Aims. Community Mental Health Teams (CMHTS) are now the cornerstone of modern mental health care and play a central role in assessment, diagnosis and care coordination. CMHTs vary widely in their service provision and composition. Within teams there is latitude for variation of professional roles but the extent to which different disciplines undertake generic and profession-specific work is poorly defined. This cross-sectional study aims to establish how professional training influences the distribution of case-load mix within a general adult CMHT
Method. The GR1 CMHT provides care to a mixed urban/rural population of 25,000 in Galway city and Connemara. A review was conducted of multi-disciplinary case notes for all patients actively registered with the team for a period of one year. Name, age, gender, whether referred or admitted in the past year, medication and day hospital attendance were recorded. Clinical diagnoses were recorded but, where missing, verified with a relevant team member. The team consultant reviewed and verified the $1 \mathrm{CD}-10$ primary clinical diagnosis for all patients. Evidence of clinical input by multidisciplinary team members was recorded from clinical files with the final electronic database being checked by each professional for accuracy. We examined any input over the past year rather than

frequency of input. Patient characteristics and diagnosis by professional discipline were examined using descriptive statistics. Result. Of a total of 246 patients registered to the team, $37.8 \%$ (N = 93) saw one, $34.6 \%(\mathrm{~N}=85)$ saw two and $24.4 \%(\mathrm{~N}=60)$ saw 3 or more team members. Of those who saw three or more team members, psychotic disorders represented the majority diagnoses $(40 \%, \mathrm{~N}=24)$ followed by personality disorders $(25 \%, \mathrm{~N}=15)$ and affective disorders $(15 \%, \mathrm{~N}=9)$. Patients were most commonly seen by a doctor $(91.5 \%, \mathrm{~N}=225)$ followed by community mental health nurses (CMHNs) $(52.8 \%, \mathrm{~N}=130)$. Doctors saw $85 \%$ or more of all patients grouped by ICD-10 diagnoses. The majority of social work and occupational therapy case-mix comprised psychotic disorders ( $\mathrm{SW}=44.2 \%$, OT $=34.2 \%$ ) followed by personality disorders $(\mathrm{SW}=25.6 \%$, OT $=23.7 \%)$. Of psychology case-mix, the highest was personality

disorders at $41.6 \%(\mathrm{~N}=13)$ followed by anxiety and related disorders at $25 \%(\mathrm{~N}=8)$. CMHN case-mix was highest for psychotic disorders at $44.6 \%(\mathrm{~N}=58)$ followed by $21.5 \% \operatorname{mood}$ disorders $(\mathrm{N}=28)$.

Conclusion. This cross sectional survey informs how we currently target our specialist resources. We will now develop this to include frequency of contact to inform resource allocation and skill mix.

\section{Mental health clothing bank - adDressing the issue Louisa Ward}

Worcestershire health and care NHS trust

doi: 10.1192/bjo.2021.604

Aims. We often have patients who are admitted to the ward wearing only the clothes they came in. These patients have no way of going to get more clothes due to being detained, poverty/ homelessness or covid restrictions. Many do not have friends or family who can bring them clothes. As such they might wear one set of clothes for a number of weeks which is bad for their physical and mental health. We are creating a clothes bank to provide a change of clothes for these patients, and help their recovery back into the community. Many have clothes that are inappropriate for the current weather, or do not have a set of smart enough clothes for a job interview. We feel that this simple intervention will have a big community impact.

Method. We have obtained support from a number of charities and companies to supply donations. The project will be led by a team of staff and patients.

Result. We will review the usage of this scheme in 6 months time Conclusion. We hope this intervention will tackle the issue of clothing on mental health wards. In the future we wish to expand this to outpatient mental health service users. We would then like to expand this project countrywide as are unaware of any other areas providing something similar. 